\title{
Targeted tissue perfusion versus macrocirculation-guided standard care in patients with septic shock (TARTARE-2S): study protocol and statistical analysis plan for a randomized controlled trial
}

\author{
Ville Pettilä ${ }^{12^{*}}$, Tobias Merz ${ }^{1}$, Erika Wilkman², Anders Perner ${ }^{3,5}$, Sari Karlsson ${ }^{4}$, Theis Lange ${ }^{5,6}$, Johanna Hästbacka ${ }^{2}$,
} Peter Buhl Hjortrup ${ }^{3}$, Anne Kuitunen ${ }^{4}$, Stephan M. Jakob ${ }^{1}$ and Jukka Takala ${ }^{1}$

\begin{abstract}
Background: Septic shock has a 90-day mortality risk of up to $50 \%$. The hemodynamic targets, including mean arterial pressure (MAP) are not based on robust clinical data. Both severe hypotension and high doses of vasopressors may be harmful. Hence, re-evaluation of hemodynamic targets in septic shock is relevant.

Methods/design: The targeted tissue perfusion versus macrocirculation-guided standard care in patients with septic shock (TARTARE-2S) trial is a prospective, two-parallel-group, randomized, open-label, multicenter trial with assessor-blinded outcome evaluation. We will randomize at least 200 patients with septic shock in four European intensive care units (ICUs) to test whether a tissue perfusion-guided treatment strategy based on capillary refill time, peripheral temperature, arterial lactate concentrations, and accepting lower MAP levels, leads to a faster resolution of shock than macrocirculation target-guided standard care.

The primary outcome measure is days alive in 30 days with normal arterial blood lactate (first value of $<2 \mathrm{mmol} / \mathrm{L}$ ) and without any inotropic or vasopressor agent. Secondary outcomes include individual components of the primary outcome, days alive without renal replacement, days alive without mechanical ventilation in 30 days, and new acute kidney injury. The sample size enables detection of a 13.5-h difference in the primary outcome with a type 1 error of $5 \%$ and power of $80 \%$, assuming $25 \%$ mortality and a mean of $650 \mathrm{~h}$ (SD 30) among the 30-day survivors. After 150 included patients the statistician masked for allocation group will recalculate the sample size potentially increasing the sample up to 300. The Data Safety and Monitoring Board (DSMB) will review the safety data after 100 patients.
\end{abstract}

Discussion: The TARTARE-2S trial will provide important clinical data on treatment targets in septic shock, evaluating the impact of clinical tissue perfusion-guided hemodynamic treatment on a surrogate outcome combining resolution of shock (hyperlactatemia and vasopressors/inotropes), and 30-day mortality.

Trial registration: ClinicalTrials.gov: NCT02579525. Registered on 19 October 2015.

Keywords: Septic shock, Critical illness, Tissue perfusion, Lactate, Vasopressor, Mortality

\footnotetext{
* Correspondence: ville.pettilae@insel.ch

1 Department of Intensive Care Medicine, Bern University Hospital (Inselspital), University of Bern, Bern, Switzerland

${ }^{2}$ Division of Intensive Care Medicine, Department of Perioperative, Intensive

Care and Pain Medicine, University of Helsinki and Helsinki University

Hospital, Helsinki, Finland

Full list of author information is available at the end of the article
} 


\section{Background}

Septic shock affects millions of people annually and has a 90-day mortality rate of 18 to $50 \%$ [1-5] depending on the patient population and study selection criteria. Early hemodynamic resuscitation and stabilization is considered to be crucial for improved survival. Current resuscitation strategies aim to achieve hemodynamic stabilization as early as possible in order to prevent subsequent organ dysfunction. The guidelines for initial hemodynamic resuscitation of septic shock use primarily macrocirculatory targets, such as mean arterial blood pressure (MAP) and central venous pressure (CVP), and surrogate markers of tissue perfusion such as diuresis. Attempts to use central venous or mixed venous oxygen saturation $\left(\mathrm{ScVO}_{2}, \mathrm{SvO}_{2}\right)$ to guide resuscitation have been disappointing. Despite early enthusiasm based on a small single-center trial which suggested a major improvement in survival [6], no benefit of $\mathrm{ScVO}_{2}$ guided treatment was found in three large multicenter trials $[1,4,5]$.

The current SSC guidelines (Surviving Sepsis Campaign: International Guidelines for Management of Severe Sepsis and Septic Shock: 2012) [7] still emphasize a MAP target and to a lesser extent tissue perfusion. Systemic blood pressure and flow and microcirculatory blood flow do not correlate well in septic shock. This is especially true if systemic blood pressure is achieved with vasoconstrictive drugs which per se may impair microcirculation and clinical outcome [8]. Furthermore, a recent systematic review [9] underscored the paucity of clinical evidence to guide the use of vasopressors and to support the current recommendations regarding MAP target levels. In summary, the current resuscitation guidelines for septic shock might direct physicians to use higher doses of vasopressors and inotropes and, thus, facilitate iatrogenic aggravation of shock-induced tissue hypoperfusion.

Impaired tissue perfusion and microcirculation are considered to be hallmarks of septic shock, and increased arterial blood lactate in septic shock is a marker of poor prognosis. These concepts provide a rationale for therapeutic strategies focusing more on improvement of tissue perfusion than systemic hemodynamics. Normalization of elevated blood lactate levels has been proposed to guide resuscitation in septic shock because it may reflect tissue perfusion during shock resuscitation better than strategies based mainly on macrohemodynamic targets. The duration of elevated lactate levels is a clinically relevant surrogate endpoint previously related to robust patientrelated adverse outcomes, such as multiple organ dysfunction [10] and mortality [11, 12]. In addition, lactate is also associated with peripheral perfusion
[13], and has been used as a target in a previous large study in septic shock in comparison to $\mathrm{ScVO}_{2}$ targeted therapy [14]. Abnormal peripheral perfusion, defined as arm-to-fingertip temperature gradient of $>4{ }^{\circ} \mathrm{C}$, and capillary refill time $(\mathrm{CRT})>4.5 \mathrm{~s}$ ), is associated with multiple organ dysfunction in critically ill patients [15] and with impaired tissue oxygen saturation and poor outcome in septic shock patients [16]. Of note, subjectively assessed warmness of the peripheral skin has good agreement with the measured temperature differences [15]. The mottling score [17], reflecting perfusion of the knee skin area, has also been used. Additionally, biomarkers (to be explored in sub-studies) indicating endothelial or cardiac damage could elucidate the potential mechanisms leading to untoward clinical patient-related outcomes, such as septic acute kidney injury (AKI) [18] treated with renal replacement therapy (RRT) [19], multiple organ dysfunction, and death.

\section{Aim}

The objective is to compare the feasibility and the effect of resolution of shock of two approaches to the management of tissue hypoperfusion in septic shock: (1) targeted tissue perfusion (TTP) approach versus (2) macrocirculation-guided (MCG) care - the latter reflecting recommended standard care.

\section{Methods/design}

This trial is a prospective, investigator-initiated, multicenter, two-parallel-group, randomized, open-label, multicenter trial of TTP versus MCG care in patients with septic shock (Appendix 1) with adequate generation of allocation sequence, and adequate allocation concealment. Patients will be stratified by center and by the presence/absence of chronic hypertension. Each participating unit will go through an educational program regarding study monitoring methods (including CRT), and study reporting methods (including repeated hemodynamic target assessments and treatment attempts).

The recruitment begins after approval from local ethics committees.

\section{Ethics committee approvals submission/approval}

Bern University Hospital Ethics Committee (Kantonal Ethiko Kommittee (KEK) Bern) 19 October 2015/22 March 2016.

Helsinki University Hospital Operative Ethics Committee - 23 October 2015/16 December 2015.

Rigshospitalet Ethics Committee - April 2016/ pending. 


\section{Hypothesis}

We hypothesize that targeting clinical tissue perfusion (the TTP arm) will decrease the use and untoward effects of vasopressors, and result in more days alive in 30 days with normal arterial blood lactate (first value of $<2 \mathrm{mmol} / \mathrm{L}$ ) and without any inotropic or vasopressor agent - compared to standard clinical care with preference of macrocirculatory targets (the MCG arm).

\section{Trial interventions}

All patients will be treated according to the targets (Appendix 2) of the allocated arm:

1. Intervention group - targeted tissue perfusion (TTP) care

2. Control group - macrocirculatory targets-guided (MCG) standard care

All interventions in both groups will be given at the discretion of the treating clinicians according to the targets. Both the hemodynamic problems detected and the given interventions will be registered at each change of treatment over time (Appendices 3 and 4).

\section{Concomitant interventions}

Treatment of septic shock is complex with multiple interventions [6] and, as blinding of treating personnel is not feasible, the use of several concomitant interventions may be influenced by the allocated intervention arm. In order to minimize these potential differences, treatment suggestions for the following interventions will be provided:

- Vasopressors - norepinephrine highly recommended (the hemodynamic problems to be registered and reported Appendix 3)

- Fluids - correction of hypovolemia (preferably crystalloids, starch not to be used) will be at the discretion of the treating clinician (Appendix 3)

- Avoidance of excess fluids after $6 \mathrm{~h}$ from randomization (amount of given fluids and balance over time up to $72 \mathrm{~h}$ will be registered and reported)

- Inotropic agents - to increase impaired flow, dobutamine is preferred - if ineffective, adrenaline may be used

- Glucocorticoids - recommended not to be used

- Blood products - red blood cell (RBC) transfusion trigger $70 \mathrm{~g} / \mathrm{L}$, unless ischemia or active bleeding [2]
- Renal replacement therapy (RRT) suggested criteria according to conventional standard criteria $[19,20]$, with proven feasibility

[21] as follows:

(a) serum potassium $\geq 6.0 \mathrm{mmol} / \mathrm{L}$, or

(b) $\mathrm{pH}<7.20$ and serum bicarbonate $\leq 10 \mathrm{mmol} / \mathrm{L}$, or

(c) evidence of severe respiratory failure, based on a $\mathrm{PaO}_{2} / \mathrm{FiO}_{2}<200$ and clinical perception of volume overload and oliguria, or

(d)persistent severe AKI (serum creatinine remains $>50 \%$ the value recorded at randomization) for more than $72 \mathrm{~h}$ from randomization

- Lung-protective ventilation - positive endexpiratory pressure (PEEP) $\geq 5 \mathrm{cmH}_{2} \mathrm{O}$, tidal volume $<8 \mathrm{ml} /$ ideal body weight, and plateau pressure $<30$ $\mathrm{cmH}_{2} \mathrm{O}$

\section{Inclusion criteria}

Septic shock defined as (Appendix 1):

1. Infection (suspected or documented) and

2. Systemic mean blood pressure above $65 \mathrm{mmHg}$ requiring any dose of vasopressors (norepinephrine, epinephrine, vasopressin) despite adequate fluid resuscitation (minimum of $20 \mathrm{ml} / \mathrm{kg}$ (actual body weight) crystalloids) and

3. Elevated lactate $\geq 3.0 \mathrm{mmol} / \mathrm{L}$ with suspected hypoperfusion

\section{Exclusion criteria}

- Age below 18 or over 80 years

- Any other probable condition than sepsis affecting or expected to affect the central nervous system, including post cardiac arrest

- Myocardial ischemia

- Acute pulmonary embolism

- Terminal illness and not considered for full intensive care support

- Use of extra-corporeal membrane oxygenation (ECMO)

- Known liver disease - Child-Pugh classes B or C

- Known chronic kidney disease

- Known to be pregnant or lactating

- More than $4 \mathrm{~h}$ from fulfilled inclusion criteria (to be fullfilled at the latest 4 hours after ICU admission) to randomization (to be done in 8 hours from ICU admission)

- Other probable cause of hyperlactatemia

- Patients transferred from another intensive care unit (ICU)

- Patients with active hematological malignancy 


\section{Randomization}

Randomization will be done using a computer-based algorithm created by an independent statistician, to allow immediate and concealed allocation to the intervention arm. The patients will be stratified according to the site and presence/absence of chronic hypertension (with known medication). A varying block size will be used. Each patient will be allocated a unique patient ID-number. Randomization is targeted to be performed within $2 \mathrm{~h}$ after fulfillment of the inclusion criteria in the ICU (but no later than $4 \mathrm{~h}$, the fulfillment of which is an exclusion criterion).

\section{Primary outcome measure}

- Days alive in 30 days - with normal arterial blood lactate (first confirmed value of $<2 \mathrm{mmol} / \mathrm{L}$ and without any inotropic or vasopressor agent)

\section{Secondary outcome measures}

1. Time to normalization of lactate

2. Days alive with normal lactate (all values $<2 \mathrm{mmol} /$ L) in 30 days

3. Days alive without the use of inotropic or vasopressor agents in 30 days

4. Days alive without RRT in 30 days

5. Days alive without mechanical ventilation in 30 days

6. Days alive without any organ support (mechanical ventilation, RRT) in 30 days

7. New AKI according to the KDIGO classification (stages I-III)

8. 8.. Days alive outside hospital in 90 days

9. Total amount of norepinephrine given until day 5

10.Number/total number of the following adverse reactions:

(a) ventricular tachycardia/fibrillation

(b)atrial fibrillation

(c) myocardial infarction

(d)skin necrosis

(e) stroke

(f) secondary bowel ischemia

(g) limb ischemia

(h)total numbers of serious adverse reactions (SAR) (numbers of patients and reactions)

\section{Exploratory outcomes}

All-cause mortality at day 90 .

\section{Blinding}

Blinding of health care providers will not be feasible which infers that all clinical staff caring for the patients will be aware of the allocation during the intervention period. The two interventions may lead to different use of concomitant interventions, but the lack of blinding may also result in differences in the use of concomitant interventions during the intervention period. Hence, we will provide suggestions for the use of relevant cointerventions (see "Concomitant interventions") and will record the use of them.

Information on the primary outcome and other secondary outcomes will be provided by the local investigators from patient charts, but the statistician doing the analyses will be blinded to which intervention the patients received. Information on whether the exploratory outcome of death occurs will be acquired through public registers (the National Civil Registries) without knowledge of which intervention group the patient was allocated to. In Switzerland, the patient or relatives will be contacted. The assessor of the outcomes will be blinded to the study group. The members of DSMB will remain blinded unless they request otherwise.

\section{Participant discontinuation and withdrawal}

Patients who are withdrawn from the trial protocol will be followed up and analyzed as the remaining patients (intention-to-treat analysis, ITT).

\section{Suspension of the protocol}

The protocol may temporarily be suspended for the individual patient, at the discretion of the treating clinicians, if the patient is to be resuscitated in the presence of any acute condition superimposed on septic shock, as judged by the investigator. Patients to be operated on at operational theatres may also have their procedures suspended during that time.

\section{Severe adverse reactions (SARs)}

SARs to norepinephrine:

1. Cerebral hemorrhage seen on computed tomography $(\mathrm{CT})$ or magnetic resonance imaging (MRI) scan

2. Cardiac arrhythmia resulting in the use of medication or electrical cardioversion

3. Psychiatric symptoms resulting in the use of antipsychotic drugs

Not registered as SARs to norepinephrine: tremor, headache, dizziness, and sweats are not registered. Hypertension is not registered. Dyspnea itself is not 
regarded as a serious adverse event (SAE). Hypersalivation, nausea, and vomiting are not registered.

SAEs will not be recorded as an entity, because the majority of septic ICU patients will experience several SAEs during their critical illness. The most important SAEs will be captured in the secondary outcome measures and in the daily Sequential Organ Failure Assessment (SOFA)-scoring. Patient charts will contain daily registrations of detailed clinical data, which can be obtained on request from the medical authorities. Trial investigators are to report suspected unexpected serious adverse reactions (SUSARs) without undue delay to the chief investigators, which in turn will report these to the Swiss, Finnish and Danish Health and Medicine Authorities within 7 days after the report has been received.

\section{Statistical plan and data analysis Sample size and power}

We assume $25 \%$ mortality in both study groups, and this phase II trial will be unlikely to have the power to detect any mortality difference between the intervention groups. Therefore, a surrogate endpoint (days alive in 30 days with normal lactate and without vasopressors) combining survival, need for vasopressor therapy, and normalization of hyperlactatemia has been chosen. A post-hoc analysis from the FINNAKI study in a similar study population revealed a 30-day mortality rate of $25 \%$, and in 98 patients (of 128) who survived the mean time alive without vasopressors or hyperlactatemia within 30 days $(720 \mathrm{~h}$ ) was $650 \mathrm{~h}$ (SD 35) (unpublished data).

In the power analysis we assumed that the time with vasopressors or hyperlactatemia among the 30-day survivors follow a log-normal distribution with mean of $70 \mathrm{~h}$ (70 minus "treatment effect among survivors for the TTP group") and SD 30, this simulated value was subtracted from $720 \mathrm{~h}$ (the number of hours in 30 days). For each configuration of sample size, mortality rate and expected treatment effect we simulated data and compared the two groups using a Mann-Whitney test. Ten thousand simulations were done for each configuration to compute the power. The required sample sizes (both groups combined) to achieve $80 \%$ power as a function of mortality proportion (i.e., fraction of patients with a zero value for the primary endpoint) and average change in the primary endpoint among survivors are displayed in Table 1.

The clinically relevant difference in the study primary outcome to be tested is $18 \mathrm{~h}$ (Amendment 1 to the study protocol -26 October 2015). An adaptive sample size adjustment will be employed. After 150 patients have been included and followed for 30 days, the study statistician (Assoc. Professor T. Lange, University of Copenhagen) will redo the power calculation based on the observed mortality proportion and the observed dispersion in the primary outcome. The calculation will be done without un-blinding the study and will follow the previous recommendation by Chow and Chang (based on the revised power calculation a suggestion for a revised sample size will be presented to the investigators) (https://www.crcpress.com/AdaptiveDesign-Methods-in-Clinical-Trials-Second-Edition/ChowChang/9781439839874).

\section{Statistical methods}

The study flowchart is included as Fig. 1. A MannWhitney test for differences in continuous outcomes including the primary outcome measure, and Fisher's exact test for dichotomous variables will be done as a primary analysis. A sensitivity analysis in subgroups of patients with and without previous hypertension will be performed. The components of the primary outcome measure (time to normalization of lactate, time to stopping of all vasoactive drugs, and day-30 mortality) are all included among secondary outcomes. In all tests a $p$ value of less than 0.05 will be considered statistically significant. If missing data are $>5 \%$, multiple imputations will be performed. A detailed statistical analysis plan is provided in Appendix 5.

Table 1 Power calculations

\begin{tabular}{llll}
\hline \multirow{2}{*}{ Mortality proportion } & \multicolumn{2}{l}{ Change among survivors in hours (and among all patients) } \\
\cline { 2 - 4 } & $12 \mathrm{~h}(9 \mathrm{~h})$ & $18 \mathrm{~h}(13.5 \mathrm{~h})$ & $24 \mathrm{~h}(18 \mathrm{~h})$ \\
\hline $20 \%$ & 340 & 156 & 90 \\
$25 \%$ & 438 & 194 & 118 \\
$30 \%$ & 562 & 270 & 158 \\
$35 \%$ & 660 & 348 & 210 \\
$40 \%$ & 660 & 456 & 282 \\
\hline
\end{tabular}




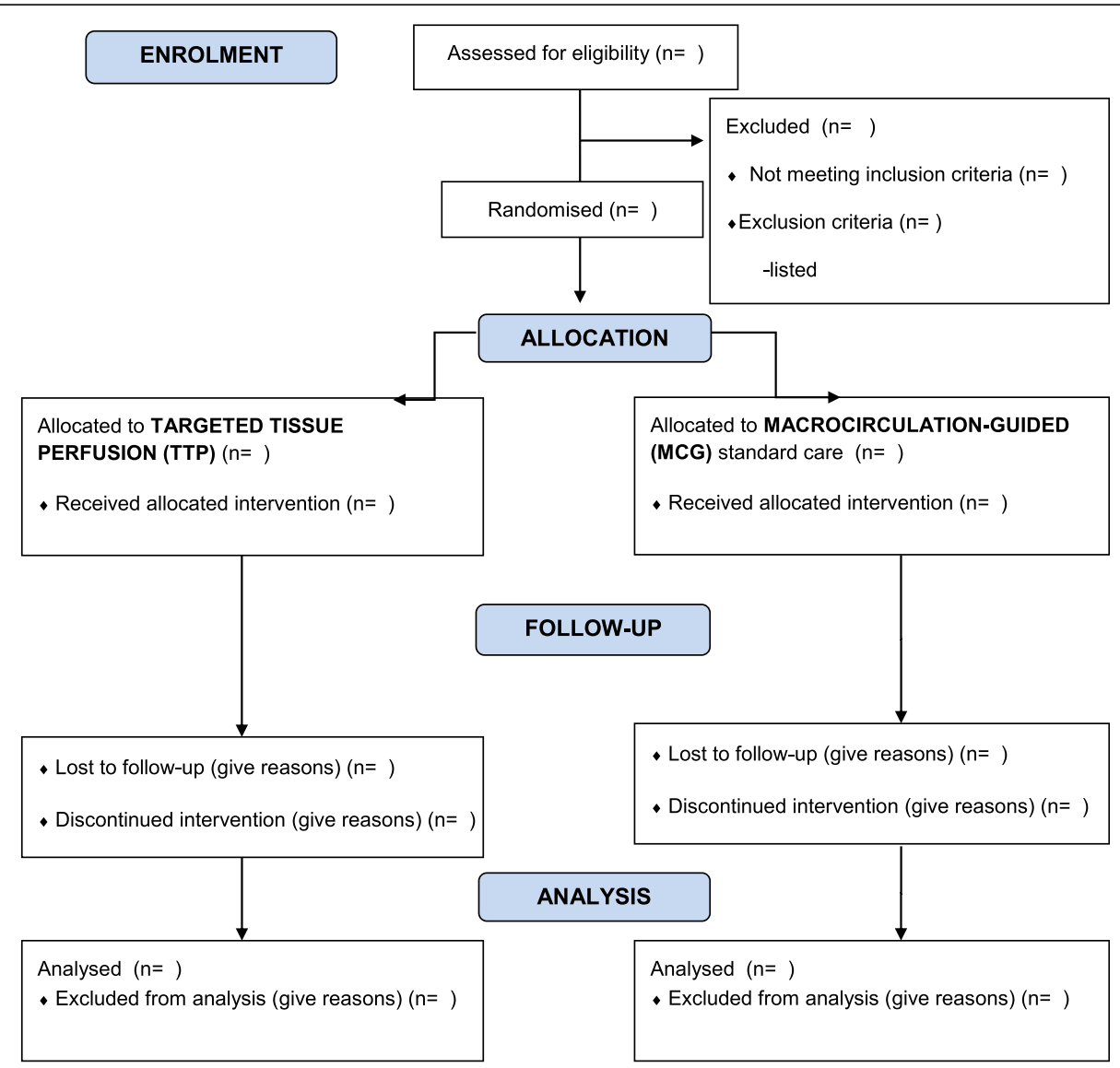

Fig. 1 The TARTARE-2S study flowchart

\section{Interim analysis}

As this trial is a feasibility trial we do not consider an interim analysis appropriate. As an interim analysis will not be performed, statistical early stopping criteria will not be applied. However, the Data Safety and Monitoring Board (DSMB) has full access to the safety data and will do a thorough review of these after 100 patients, and may recommend stopping the study anytime based on safety concerns.

\section{Intervention accountability}

Every patient will be allocated a registration sheet to be kept in the site master file. The initials, birth date, screening number, time for randomization, and study arm will be included. The compliance of trial arm targets for each study patient will be checked hourly for the first $72 \mathrm{~h}$, and clinical problems and given hemodynamic treatments will be registered until the study combined endpoint has been fulfilled, up to day 30. The originals for these documents will be kept as the study source data at each site.

\section{Registration}

ClinicalTrials.gov: NCT02579525 (Registration date 19 October 2015).

\section{Data to be registered}

The continuous data on hemodynamic parameters will be registered as 10 -min medians using electronic PMDS, when possible and otherwise registered each hour to 72 h. For other data please see "Appendix 4." The Standard Protocol Items: Recommendations for Interventional Trials (SPIRIT) figure regarding study interventions and timing is provided as Fig. 2.

\section{Data handling}

Data will be entered into an electronic, web-based electronic Case Report Form (eCRF) from patient notes by trial personnel. Each patient will receive a unique trial identification number. Trial investigators will receive a personal username and passwords to access the TARTARE-2S trial web-page. Each site will only have access to site-specific data. Data will 


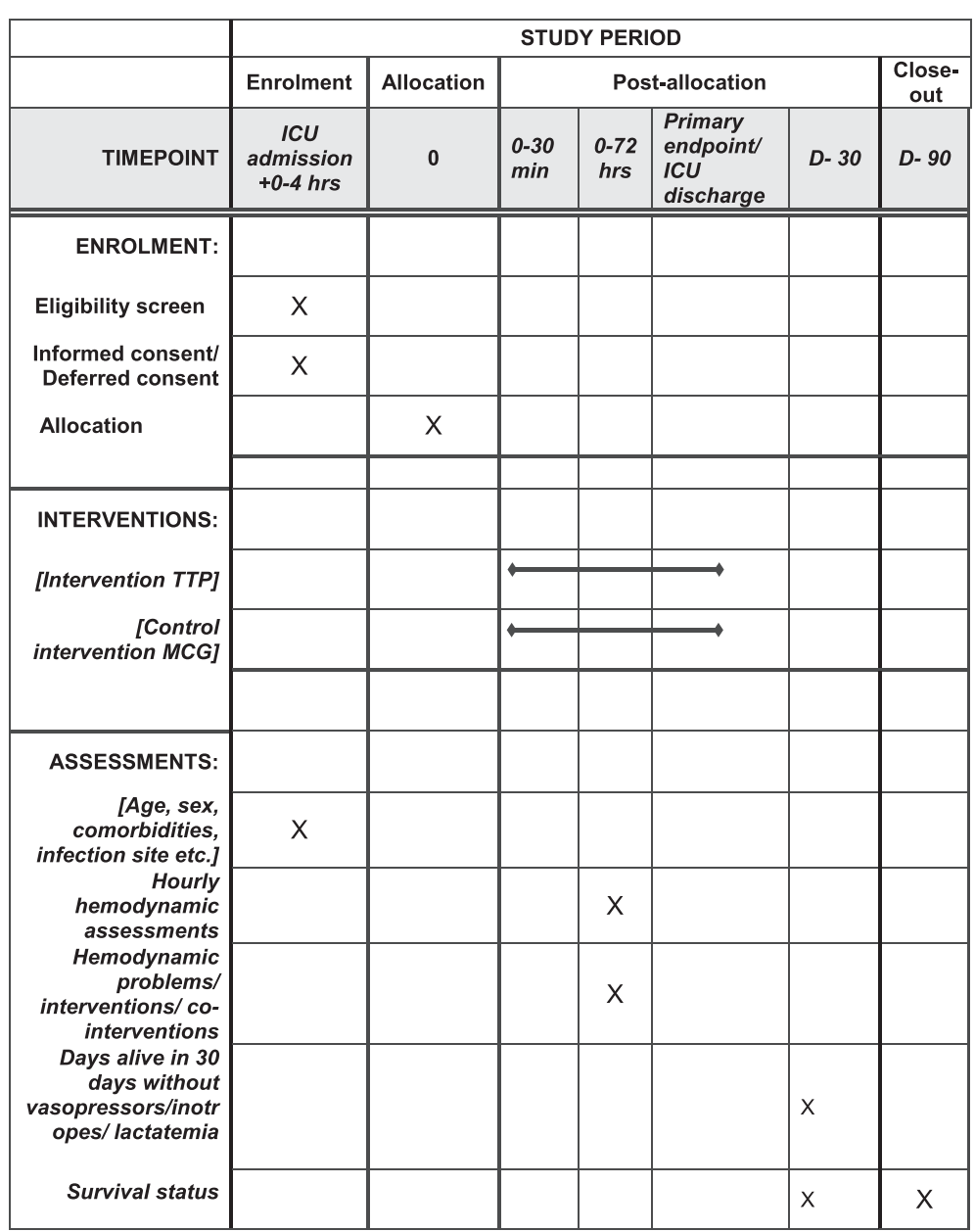

- TTP- Targeted tissue perfusion group; MCG- Macrocirculatory-guided control group

Fig. 2 The schedule of enrollment, interventions, and assessments

be handled according to the Swiss, Finnish and Danish laws. All original records (including Informed Consent Forms, eCRFs, and relevant correspondences) will be archived at trial sites for 15 years. The clean electronic trial database file will be delivered to the Inselspital Data Archive and maintained for 15 years and anonymized, if requested by the authorities.

\section{Monitoring}

The trial will be externally monitored (Clinical Trial Unit, Bern) to Good Clinical Practice (GCP) standards. A centralized day-to-day monitoring of the eCRF will be done by the coordinating investigator.

\section{Ethical considerations}

In addition to fluid therapy, norepinephrine administration to increase the MAP is a key element in the treatment of septic shock. As the intervention is very frequent, but not evidence-based and carries potential risks, we consider it to be in the wider interests of society and patients to perform research in this area, as clearly highlighted in a recent systematic review and meta-analysis [9]. The present trial is a phase II trial, i.e., a trial assessing both the feasibility of the protocol in a clinical setting and a patient-centered surrogate outcome. Should the trial prove feasible with separation between the two interventions, and suggest a benefit in terms of the primary endpoint, a larger phase III trial assessing 90-day mortality is intended. Thus, it is the opinion of the Steering Committee that this study is very important and ethically justified.

In some retrospective observational studies, low MAP has been associated with an increased risk of acute kidney injury (AKI) [22-24]. However, causal inferences cannot be drawn from observational data with the inherent limitation that the most severely ill 
patients inevitably have lower MAPs. In the present study: (1) a minimum safety limit of MAP has been set to the TTP group, (2) the study patients are carefully monitored and re-evaluated, and treatment goals are registered every hour. In addition, (3) the treating clinician should allow lower MAP values only in situations without any clinical problems, such as oliguria, in which cases individual use of higher MAP targets is allowed (and also in previously hypertensive patients). Thus, the Steering Committee has strong reasons to believe that the care of the study patients will be better and safer than the highly varying standard clinical practice; e.g., regarding fluid treatment [25]. In addition, high-quality randomized controlled trials (RCTs) focusing on unproven but widely used standard treatments have been shown to change clinical practice and to save future critically ill patients from untoward adverse events and death, e.g., by avoiding the use of starch in septic shock [26].

Treatment of septic shock is time-dependent and many patients will be unconscious and, due to fever and/or septic brain dysfunction, unable to consider an informed consent. Inclusion of only conscious less severely ill patients would cause a significant bias and jeopardize the study results. Furthermore, the treatment separation and effect are plausibly most obvious the earlier the randomization occurs. Of note, both treatment arms are within the frame of current standard clinical practice and no investigational drugs are used, thus, ethically allowing the potential use of a deferred consent. Therefore, patients will be included either with a deferred consent, or a proxy consent (next of kin) according to national laws in Finland, Switzerland, and Denmark (two-physician consent also allowed in Denmark). The patient or next of kin and/or general practitioner will be asked for a deferred consent if required by national law.

The trial will adhere to the trial protocol, the Helsinki Declaration in its latest form, GCP guidelines, and the national laws in the countries involved. Inclusion will start after approval by the ethics committees, medicines agencies, data protection agencies, and health authorities in the countries of the trial sites.

\section{Informed consent}

The process leading to obtaining informed consent will be in compliance with all applicable regulations and national laws. Patients with septic shock are seldom able to consider their consent due to fever, mechanical ventilation, sedation, and/or septic encephalopathy. However, those patients who regain consciousness will be asked for informed consent as soon as possible. The possible benefit of the treatment (and the separation between the study arms) will plausibly be larger the sooner the treatment is started. In addition, it is important not to introduce a significant bias to the study results by only including less severely ill patients because most plausibly the most severely ill patients will benefit most. The consenting party will be provided with written and oral information about the trial so they are able to make an informed decision about participation in the trial. Written information and the consent form will be subjected to review and approval by the National Ethics Committee.

\section{Duration}

For clinical treatment, primary and secondary endpoint up to 30 days; follow-up exploratory endpoint until day 90 .

\section{Co-enrollment}

Possible simultaneous co-enrollment to any other RCT will be discussed by the Management Committee.

\section{Timeline}

Trial sites determined - September 2015

Governance approval applications submitted -

October 2015

First participant enrolled - April 2016

Last participant enrolled - November 2017

Follow-up completed - December 2017

Data analysis and submission for the main publication - March 2018

Laboratory analyses completed - June 2018

Publications of clinical sub-studies and laboratory analyses completed - December 2018

\section{Trial organization \\ Steering Committee}

Ville Pettilä, Helsinki University Hospital, and Inselspital, Bern (CI1)

Jukka Takala, Inselspital, Bern, Switzerland (CI2)

Stephan Jakob, Inselspital, Bern, Switzerland

Anders Perner, Copenhagen University Hospital, Rigshospitalet, Denmark.

\section{Site principal investigators (PIs)}

Tobias Merz, Inselspital, Bern

Erika Wilkman, Helsinki University Hospital

Sari Karlsson, Tampere University Hospital

Anders Perner, Rigshospitalet, Copenhagen 
Data Safety and Monitoring Board (DSMB)

Professor Konrad Reinhart (chair), Jena

Professor Peter Jüni, Bern/Toronto

Professor Jan Wernerman, Stockholm

\section{Independent statistician}

Assoc. Professor Theis Lange, Centre for Research in Intensive Care and Section of Biostatistics, University of Copenhagen.

\section{Trial sponsors}

The coordinating investigators, CI1 Ville Pettilä and CI2 Jukka Takala, are trial sponsors.

\section{Laboratory measurements}

Ethylenediaminetetraacetic acid (EDTA) plasma samples and whole blood will be drawn as soon as possible after ICU admission, and at $72 \mathrm{~h}$ from ICU admission for later analysis of patient-related changes in biomarkers indicating endothelial/myocardial damage, such as CD73 and vascular adhesion protein-1 (VAP-1), heparin-binding protein (HBP), chromogranin A (CgA), metabolomics, and mitochondrial function tests.

\section{Publication plan}

All trial results whether positive, negative, or neutral will end up in the public domain, preferably in a peer-reviewed publication. All trial sites with at least 25 randomized patients will be granted one authorship for the site PI, and each additional 25 patients will guarantee one additional authorship for the site AIs. A trial statistician (TL) will also be granted an authorship. The order of authorships for the study main publication will be as follows: VP, SJ, and AP will be the first, second, and third, and JT the last author. Other authors will be in the order of included number of patients with high-quality data: site PIs first, and AIs thereafter. The authorships for the publications of the main and sub-studies will follow the International Committee of Medical Journals Editors' (ICMJE) principles. The full protocol and the participant-level anonymized dataset will be submitted as appendices with the main paper to be publicly available after the study publication. The SPIRIT checklist for clinical trial protocol is provided as Additional file 1.

\section{Perspectives}

Septic shock affects millions of patients worldwide annually. The results of study will - in any case have a major influence on the clinical management/ international guidelines regarding treatment of septic shock. If supporting our hypothesis of the beneficial effects of TTP, the current recommendations will have to be changed leading to major scientific and societal impacts on heath and health care costs. In case MCG proves to be better, the study results will for the first time confirm the widely recommended minimum MAP level. Given that several different pathophysiological pathways are probably in interaction and may only partly explain the clinical course of the disease, the potential strength of measuring several biomarkers/mitochondrial function/ gene expression and protein synthesis in the sub-studies of this phase II study, will give us the opportunity to compare their relative importance and predictive power with regard to organ dysfunction.

\section{Discussion}

Conduct of the TARTARE-2S trial is in broad agreement with the international guidelines [7] regarding the control group. The targeted patient population fulfills the recent Sepsis-3 septic shock definition [27], representing the most severely ill septic shock patients with plausibly the highest possible chance to show a difference between the intervention arms [28], if such a difference exists. The experimental approach is supported by a recent review [9] highlighting the paucity of evidence to support the guidelines and additional evidence suggesting the relevance of more detailed monitoring of peripheral perfusion $[13,14]$ to guide the treatment in septic shock. The TARTARE-2S trial may be one of the first trials to bridge the gap between evidence and clinical practice and to provide detailed data on efficacy and safety of TTP to guide treatment in patients with septic shock.

\section{Trial status}

Patient recruitment has started in May 2016. Ethics and hospital approvals have been granted in Finland, and in Switzerland, and have been applied in Denmark (as of July 28, 2016).

\section{Appendix 1 The trial criteria for septic shock}

Septic shock is defined as:

1. Infection (suspected or documented) and

2. Systemic mean blood pressure above $65 \mathrm{mmHg}$ requiring any dose of vasopressors (norepinephrine, vasopressin) despite adequate fluid resuscitation (minimum of $20 \mathrm{ml} / \mathrm{kg}$ (actual body weight) crystalloids) and

3. Elevated lactate $\geq 3.0 \mathrm{mmol} / \mathrm{L}$ with suspected hypoperfusion 


\section{Appendix 2}

Table 2 The trial targets for the treatment arms. (Register hourly up to $24 \mathrm{~h}$ (every $2 \mathrm{~h}$ thereafter until study endpoint using tick boxes for targets)

\begin{tabular}{|c|c|}
\hline \multicolumn{2}{|c|}{ I. Intervention group - targeted tissue perfusion (TTP) care: } \\
\hline \multicolumn{2}{|l|}{ Primary targets } \\
\hline $\begin{array}{l}\text { Capillary refill time (CRT)/ } \\
\text { every hour }\end{array}$ & $<3 \mathrm{~s}$ \\
\hline $\begin{array}{l}\text { Skin mottling [17]/every } \\
\text { hour }\end{array}$ & Absent \\
\hline $\begin{array}{l}\text { Peripheral temperature/ } \\
\text { every hour }\end{array}$ & Warm \\
\hline Urine output/every hour & $\geq 0.5 \mathrm{~mL} / \mathrm{kg} / \mathrm{h}$ \\
\hline Arterial lactate [7]/per $2 \mathrm{~h}$ & $<2.0 \mathrm{mmol} / \mathrm{L}$ \\
\hline Mean arterial pressure (MAP) & $50-65 \mathrm{mmHg}$ (minimum as a safety limit) \\
\hline alf previous hypertension [7] & ${ }^{\mathrm{a}} 65-70 \mathrm{mmHg}$ \\
\hline \multirow[t]{2}{*}{ blf oliguria $<0.3 \mathrm{ml} / \mathrm{kg}$ [7] } & b2-h trial 75-80 mmHg, \\
\hline & $\begin{array}{l}\text { If diuresis improves, continue for } 2 \mathrm{~h} \text { and } \\
\text { re-evaluate }\end{array}$ \\
\hline \multicolumn{2}{|l|}{ Secondary target } \\
\hline $\begin{array}{l}\text { Continuous } \mathrm{SvO}_{2}[7] \text {, if } \\
\text { available }\end{array}$ & $>65 \%^{\mathrm{c}}$ \\
\hline \multicolumn{2}{|c|}{ II. Control group - macrocirculatory targets-guided (MCG) standard care } \\
\hline \multicolumn{2}{|l|}{ Primary targets } \\
\hline $\begin{array}{l}\text { Mean arterial pressure (MAP) } \\
\text { [7] }\end{array}$ & $65-75 \mathrm{mmHg}$ \\
\hline alf previous hypertension [7] & ${ }^{\mathrm{a}} 75-80 \mathrm{mmHg}$ \\
\hline \multirow[t]{2}{*}{ b lf oliguria $<0.3 \mathrm{ml} / \mathrm{kg}$} & b2-h trial 85-90 mmHg \\
\hline & $\begin{array}{l}\text { If diuresis better, continue for } 2 \mathrm{~h} \text { and } \\
\text { re-evaluate }\end{array}$ \\
\hline \multirow{2}{*}{$\begin{array}{l}\text { Central venous pressure } \\
\text { (CVP) [7] }\end{array}$} & $8-12 \mathrm{mmHg}$ \\
\hline & $\begin{array}{l}\text { Adequate fluid therapy is indicated to } \\
\text { restore clinical hypovolemia up to the } \\
\text { recommended CVP level of } 8-12 \mathrm{mmHg} \text {, } \\
\text { if needed }\end{array}$ \\
\hline Urine output [7] & $\geq 0.5 \mathrm{~mL} / \mathrm{kg} / \mathrm{h}$ \\
\hline \multicolumn{2}{|l|}{ Secondary target } \\
\hline $\begin{array}{l}\text { Continuous } \mathrm{SvO}_{2}[7] \text {, if } \\
\text { available }\end{array}$ & $>65 \%^{\mathrm{c}}$ \\
\hline
\end{tabular}

Dellinger et al. [7] according to the SSCG - Surviving Sepsis Campaign Guidelines: MAP, CVP, diuresis, $\mathrm{SvO}_{2}-1 \mathrm{C}$, lactate $-2 \mathrm{C}(1-$ a recommendation, 2 - a suggestion, $C$ - low level of evidence)

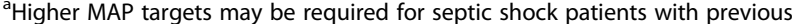
hypertension; ${ }^{\mathrm{b}}$ and a test of providing higher MAP target for $2 \mathrm{~h}$ is recommended for those with oliguria

${ }^{\mathrm{b}}$ The treating physicians should target to the lowest possible vasopressor use to maintain the highlighted lowest possible MAP level in each treatment arm; however, allowing individual higher MAP targets with specific reasons 'Measuring of $\mathrm{ScVO}_{2}$ is not recommended $[1,4,5]$. If monitoring is clinically required, use of a pulmonary artery catheter (PAC) is recommended. Pulse continuous cardiac output (PICCO) may be used for thermodilution cardiac output measurements

\section{Appendix 3}

Table 3 Data to be gathered regarding each hemodynamic target and treatment decision/change. Indicate when treatment start or change (at the same time)

(a). Hemodynamic problem(s)

Hemodynamic problem(s)

Hypovolemia

Hypervolemia

Inadequate flow/cardiac index (Cl)

Tachycardia

Inadequate contractility

Inadequate afterload/hypotension

Excessive afterload/hypertension

Excessive vasopressor dose

(b). Given treatment(s)

Treatment(s) given

Volume

Diuretics

Inotrope

Inotrope decrease

Inotrope increase

Vasopressor increase

Vasopressor decrease

Beta-blocking agent

Vasodilating agent

\section{Appendix 4 Data to be registered}

Data will be obtained in eCRFs from a combination of national registers and medical records. Bedside target list for treatment reasons and decisions will be implemented in the PDMS $(*)$ at each site, if possible.

Baseline variables

- National identification number (if available)

- Sex

- Age at randomization

- Estimated height

- Measured (if not possible estimated) weight

- Comorbidities? Y/N (hypertension*, chronic heart failure*, previous myocardial infarction, previous stroke, chronic obstructive pulmonary disease ${ }^{*}$,

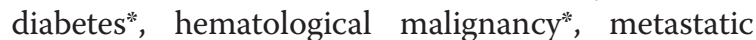
cancer*, AIDS*)

- Elective or emergency admission*

- Operative or non-operative admission* 
- Site of infection (pulmonary/abdominal/urinary tract/soft tissue/other)

- Previous plasma/serum creatinine (7-365 days before hospital admission), if available

24-h from ICU admission:

- Values for Simplified Acute Physiology Score (SAPS II)*

Twenty-four hours before randomization

- Volume of resuscitation fluids (crystalloids, colloids and blood products specified in milliliters)

- Hemoglobin (lowest)*

- SOFA score variables*

At randomization

- Norepinephrine dose and mean arterial pressure (MAP) at the same time

- Total fluids given in last $2 \mathrm{~h}$

- Lactate (last value from the previous $2 \mathrm{~h}$ )

Continuously as 10-min medians in the first $72 \mathrm{~h}$ after randomization:

- Systolic arterial pressure*

- Mean arterial pressure*

- Central venous pressure*

- Heart rate*

- Peripheral oxygen saturation $\left(\mathrm{SpO}_{2}\right)^{*}$

- Continuous central venous oxygen saturation, if available

- Continuous mixed venous saturation, if available

- Dose of norepinephrine*

- Dose of dobutamine*

One-hour intervals (for $72 \mathrm{~h}$ )

- Urinary output*

- Volume of given fluids

- CRT - only in the TTP group

- Assessment of upper arm temperature - only in the TTP group

- Assessment of skin mottling score - only in the TTP group

Two-hour intervals (for $72 \mathrm{~h}$ unless study endpoint reached)

- Arterial lactate measured in both groups

Four-hour intervals during the first $72 \mathrm{~h}$ after randomization (if not discharged from the ICU)
- Arterial blood gas tensions $\left(\mathrm{PaO}_{2}, \mathrm{PaCO}_{2}\right), \mathrm{pH}$ and base excess (BE)

- Calculated Mixed v-aCO $2 / \mathrm{Da}-\mathrm{vO}_{2}$-ratio (if pulmonary artery catheter, PAC)

- Cardiac index (if PAC)

- Stroke index (if PAC)

Daily in the first 7 days after randomization (if not discharged from the hospital):

- SOFA score variables*

- Total volume of fluids

- Estimated fluid balance

- Dose of glucocorticoids (not recommended)

Daily up to 30 days:

- On mechanical invasive- or non-invasive ventilation? Y/N

- Ventilator settings at 8 a.m. (PEEP, Pplat, Vt)

- Use of muscle relaxants during the last $24 \mathrm{~h}$ ? Y/N

- Presence of acute kidney injury (AKI) according to KDIGO classification? Y/N/and stage (I-III)

- Use of renal replacement therapy? $\mathrm{Y} / \mathrm{N}$

- Use of vasopressors? Y/N

- Use of inotropic agents? Y/N

- Ischemic events

- SARs and SUSARs

\section{Appendix 5 A detailed study statistical analysis plan}

The primary analysis of the primary outcome (as well as secondary outcomes b, c, d, e, f, h, i, and j) will be an unadjusted Mann-Whitney test. As a sensitivity analysis the $p$ values will be recalculated using permutations within groups defined by site and the presence/absence of hypertension. This test is a non-parametric version of an ANOVA adjusted for site and presence/absence of hypertension. A total of 100,000 permutations will be conducted for each test. The effect sizes will be quantified by direct comparisons of the raw means between groups. Confidence intervals will determined by nonparametric bootstrapping to accommodate the highly non-normal distribution of the outcomes.

The secondary endpoint "a" will be assessed by a Cox model treating death before normalization of lactate as a competing event. The Cox model will be adjusted for site and presence/absence of hypertension. The effect size will be quantified using the adjusted hazard ratio.

The secondary outcome "g" will be assessed using Fisher's exact test. Effect sizes will be analyzed using crude proportions along with standard normality approximation-based confidence intervals. 
The exploratory endpoint (90-day survival) will be assessed using Fisher's exact test. Effect sizes will be quantified using odds ratios from a logistic regression adjusted for site and the presence of hypertension. As a sensitivity analysis a Cox model of time to death will also be employed. The Cox model will be adjusted for site and the presence/absence of hypertension. Here, the effect size will be quantified using the adjusted hazard ratio. The assumption of proportional hazards will be assessed using Schoenfeld residuals. Finally, KaplanMeier plots will be produced.

If any of the tests presented above must be based on data with more than $5 \%$ missing multiple imputation will be employed. If just one test needs to be based on multiple imputation all tests will be conducted both as complete case analysis and multiple imputation. However, only for tests based on data with more than $5 \%$ missing will the multiple imputation approach constitute the primary analysis. The multiple imputation will be conducted in $\mathrm{R}$ using the mice package and the recommendation in accordance with recommendations in the documentation of this package. All outcome variables (secondary and primary), sex, and age as well as stratification and treatment variables will be included in the multiple imputations procedure.

In all tests a $p$ value of less than 0.05 will be considered statistically significant. It is noted that as the adaptive sample size recalculation is not depended on a particular value of the treatment effect at the time of recalculation, there is accordingly no need to adjust the significance level to take a possible adjustment of sample size into account. All analyses will be conducted in the statistical software package $\mathrm{R}$. The full statistical analysis will be conducted blinded to actual treatment allocation and a full blinded statistical report produced. Of note, un-blinding will first happen after the full statistical report has been finalized.

\section{Additional file}

Additional file 1: SPIRIT checklist. (PDF $2231 \mathrm{~kb}$ )

\section{Acknowledgements}

Funding from the Sigrid Juselius Foundation, Instrumentarium Foundation, and Helsinki University Hospital (State funding, VTR-TYH2016243) (Finland) and Bern University Hospital (Switzerland) have been received. Funding from several other foundations have been applied for. The treating physicians/ personnel will not be given money for study patients, but the trial sites will be compensated for their research nurse/coordinator work and extra costs for laboratory sampling (estimated sum $400 € /$ patient).

\section{Authors' contributions}

VP: contributed to the study design, drafted and finalized the study protocol and the manuscript, is chief investigator (Cl1), and sponsor. JT: contributed to the study design and finalizing the study protocol and revision of the manuscript, is chief investigator ( $\mathrm{Cl} 2)$, and sponsor. EW: contributed to the study design and finalizing the study protocol and revision of the manuscript. SJ: contributed to the study design and finalizing the study protocol and revision of the manuscript. TM: contributed to the study design and finalizing the study protocol and revision of the manuscript. AP: contributed to the study design and finalizing the study protocol and revision of the manuscript. SK: contributed to finalizing the study protocol and revision of the manuscript. PBH: contributed to finalizing the study protocol and revision of the manuscript. JH: contributed to finalizing the study protocol and revision of the manuscript. AK: contributed to finalizing the study protocol and revision of the manuscript. TL: Performed the sample size calculations, and the study statistical analysis plan (Appendix 5), contributed to finalizing the study protocol and revision of the manuscript. $V P, J T, E W, S J, T M, A P, S K, P B H, J H, A K$, and TL have all approved the final version of the manuscript.

\section{Competing interests}

The authors declare that they have no competing interests.

\section{Author details}

1Department of Intensive Care Medicine, Bern University Hospital (Inselspital), University of Bern, Bern, Switzerland. ${ }^{2}$ Division of Intensive Care Medicine, Department of Perioperative, Intensive Care and Pain Medicine, University of Helsinki and Helsinki University Hospital, Helsinki, Finland. ${ }^{3}$ Department of Intensive Care, Copenhagen University Hospital, Rigshospitalet, Copenhagen, Denmark. ${ }^{4}$ Department of Intensive Care, Tampere University Hospital, Tampere, Finland. ${ }^{5}$ CRIC, Center of Research for Intensive Care, Copenhagen, Denmark. 'Section of Biostatistics, University of Copenhagen, Copenhagen, Denmark.

Received: 19 December 2015 Accepted: 20 July 2016

Published online: 02 August 2016

\section{References}

1. The ARISE investigators, ANZICS Clinical Trials Group, Peake SL, Delaney A, Bailey M, Bellomo R, et al. Goal-directed resuscitation for patients with early septic shock. N Engl J Med. 2014;371:1496-506.

2. Holst LB, the TRISS Trial Group and the Scandinavian Critical Care Trials Group, Haase N, Wetterslev J, Wernerman J, Guttormsen AB, Karlsson S, et al. Lower versus higher hemoglobin threshold for transfusion in septic shock. N Engl J Med. 2014;371:1381-91.

3. Angus DC, van der Poll T. Severe sepsis and septic shock. N Engl J Med. 2013;369:840-51.

4. Investigators PCESS, Yealy DM, Kellum JA, Huang DT, Barnato AE, Weissfeld LA, et al. Protocol-based care for early septic shock. N Engl J Med. 2014;371: 1683-93.

5. Mouncey PR, Osborn TM, Power GS, Harrison DA, Sadique MZ, Grieve RD, Investigators PMIST, et al. Trial for early, goal-directed therapy in septic shock. N Engl J Med. 2015:372:1301-11.

6. Rivers E, Nguyen B, Havstad S, Ressler J, Muzzin A, Knoblich B. Early goal-directed therapy in the treatment of severe sepsis and septic shock. N Engl J Med. 2001;345:1368-77.

7. Dellinger RP, Levy MM, Rhodes A, Annane D, Gerlach H, Opal SM, et al. Surviving Sepsis Campaign: International Guidelines for Management of Severe Sepsis and Septic Shock, 2012. Intensive Care Med. 2013;39:165-228.

8. Dünser MW, Ruokonen E, Pettilä V, Ulmer H, Torgersen C, Schmittinger CA, et al. Association of arterial blood pressure and vasopressor load with septic shock mortality: a post hoc analysis of a multicenter trial. Crit Care. 2009;13:R181.

9. D'Aragon F, Belley-Cote EP, Meade MO, Lauzier F, Adhikari NK, Briel M, Group CCCT, et al. Blood pressure targets for vasopressor therapy: a systematic review. Shock. 2015;43:530-9.

10. Bakker J, Gris P, Coffernils M, Kahn RJ, Vincent JL. Serial blood lactate levels can predict development of multiple organ failure following septic shock. Am J Surg. 1996;171:2221-6.

11. Nguyen HB, Loomba M, Yang JJ, Jacobsen G, Shah K, Otero RM, et al. Early lactate clearance is associated with biomarkers of inflammation, coagulation, apoptosis, organ dysfunction and mortality in severe sepsis and septic shock. J Inflamm. 2010;7:6.

12. Nichol A, Egi M, Pettilä V, Bellomo R, French G, Hart G, et al. Relative hyperlactatemia and hospital mortality in critically ill patients: a retrospective multi-centre study. Crit Care. 2010;14:R25. 
13. Lima A, Jansen TC, van Bommel J, Ince C, Bakker J. The prognostic value of the subjective assessment of peripheral perfusion in critically ill patients. Crit Care Med. 2009;37:934-8.

14. Jones AE, Shapiro NI, Trzeciak S, Arnol RC, Claremont HA, Kline JA, Emergency Medicine Shock Research Network (EMShockNet) Investigators. Lactate clearance vs central venous oxygen saturation as goals of early sepsis therapy: a randomized clinical trial. JAMA. 2010;303:739-46.

15. Lima A, Jansen TC, van Bommel J, Ince C, Bakker J. The prognostic value of subjective assessment of peripheral perfusion in critically ill patients. Crit Care Med. 2009;37:934-8.

16. Lima A, van Bommel J, Sikorska K, van Genderen M, Klijn E, Lesaffre E, et al. The relation of near-infrared spectroscopy with changes in peripheral circulation in critically ill patients. Crit Care Med. 2011;39:1649-54.

17. Ait-Oufella H, Lemoinne S, Boelle PY, Galbois A, Baudel JL, Lemant J, et al. Mottling score predicts survival in septic shock. Intensive Care Med. 2011;37:801-7.

18. Pettilä V, Bellomo R. Understanding acute kidney injury in sepsis. Intensive Care Med. 2014;40:1018-20.

19. Smith OM, Wald R, Adhikari NK, Pope K, Weir MA, Bagshaw SM, Canadian Critical Care Trials Group. Standard versus accelerated initiation of renal replacement therapy in acute kidney injury (STARRT-AKI): study protocol for a randomized controlled trial. Trials. 2013;14:320.

20. Vaara S, Reinikainen M, Wald R, Bagshaw S, Pettilä V, The FINNAKI Group. Timing of RRT based on the presence of conventional indications. Clin J Am Soc Nephrol. 2014;9:1577-85.

21. Wald R, Adhikari NK, Smith OM, Weir MA, Pope K, Cohen A, et al. Comparison of standard and accelerated initiation of renal replacement therapy in acute kidney injury. Kidney Int. 2015;88:897-904.

22. Dünser MW, Takala J, Ulmer H, Mayr VD, Luckner G, Jochberger S, et al. Arterial blood pressure during early sepsis and outcome. Intensive Care Med. 2009;35:1225-33.

23. Poukkanen M, Wilkman E, Vaara ST, Pettilä V, Kaukonen KM, Korhonen AM, et al. Hemodynamic variables and progression of acute kidney injury in critically ill patients with severe sepsis. Data from the prospective observational FINNAKI study. Crit Care. 2013;17:R295.

24. Raimundo M, Crichton S, Syed Y, Martin JR, Beale R, Teacher D, et al. Low systemic oxygen delivery and BP and risk of progression of AKI. Clin J Am Soc Nephrol. 2015;10:1340-9.

25. Cecconi M, Hofer C, Teboul JL, Pettilä V, Wilkman E, Molnar Z, FENICE investigators and the ESICM Trial Group, et al. Fluid challenges in intensive care: The FENICE study: a global inception cohort study. Intensive Care Med. 2015:41:1529-37.

26. Perner A, Haase N, Guttormsen AB, Tenhunen J, Klemenzson G, Aneman Å Wetterslev J, 6S Trial Group and Scandinavian Critical Care Trials Group, et al. Hydroxyethyl starch 130/0.42 versus Ringer's acetate in severe sepsis. N Engl J Med. 2012;67:124-34.

27. Singer M, Deutschman CS, Seymour CW, Shankar-Hari M, Annane D, Bauer $M$, et al. The Third International Consensus Definitions for Sepsis and Septic Shock (Sepsis-3). JAMA. 2016;315:801-10.

28. Iwashyna TJ, Burke JF, Sussman JB, Prescott HC, Hayward RA, Angus DC. Implications of heterogeneity of treatment effect for reporting and analysis of randomized trials in critical care. Am J Respir Crit Care Med. 2015;192:1045-51.

\section{Submit your next manuscript to BioMed Central and we will help you at every step:}

- We accept pre-submission inquiries

- Our selector tool helps you to find the most relevant journal

- We provide round the clock customer support

- Convenient online submission

- Thorough peer review

- Inclusion in PubMed and all major indexing services

- Maximum visibility for your research

Submit your manuscript at www.biomedcentral.com/submit

) Biomed Central 